\title{
New Salts of Levofloxacin with Physiochemical, Structural and Biological Insights
}

\author{
M. Joshi ${ }^{1}$, I. Verma ${ }^{1}$, A. Gulati ${ }^{2}$, S. Rani ${ }^{3}$, C. Budhwar ${ }^{4}$, K. Raza ${ }^{5}$, A. Mukhopadhyay ${ }^{2}$, A. R. Choudhury ${ }^{1}$
}

${ }^{1}$ Department of Chemical Sciences, Indian Institute of Science Education and Research (IISER) Mohali, Sector 81, Knowledge City, S. A. S. Nagar, Manauli PO, Punjab, India. 140306, ${ }^{2}$ Department of Biological Sciences, Indian Institute of Science Education and Research, Mohali, Sector 81, S. A. S. Nagar, Knowledge City, Manauli P. O. Mohali, Punjab, India. 140306, ${ }^{3}$ MCM DAV College for Women, Sector 36, Chandigarh, Union Territory, India. 160036, ${ }^{4}$ Department of Chemistry, Indian Institute of Technology, Jodhpur, N.H. 65, Karwar, Rajasthan, India. 342037, ${ }^{5}$ Department of Pharmacy, School of Chemical Sciences and Pharmacy, Central University of Rajasthan, Bandarsindri, Dist. Ajmer, Rajasthan, India. 305817.

angshurc@iisermohali.ac.in

Crystallization of active drug molecules in the presence of various biologically acceptable molecules (acids, bases, and amino acids) with the aim of forming a new drug composite has gained immense importance in the last couple of decades [1]. This targeted cocrystallization is exercised to enhance the physical and biological properties of the APIs in the pharmaceutical industry [2]. Low aqueous solubility, poor intrinsic dissolution rate (IDR) and moisture sensitivity of the parent various drug molecules have triggered us to investigate the possibility of formation of novel salts of existing drugs for enhanced physical properties and better biological activity [3]. Levofloxacin (LFX) [4], a broad-spectrum antibiotic suffers from low aqueous solubility and poor IDR. Co-crystallization of LFX with natural organic acids has yielded novel crystalline salts of LFX. Characterization by FTIR, PXRD and DSC confirmed the formation of the new phases and single crystal X-ray diffraction data confirmed the formation of salts as well. Enhanced solubility and IDR of the resultant salts motivated us to conduct in-vitro and in-vivo biological study on selected salts. Minimum inhibitory concentration (MIC) of LFX and salts were determined in E. coli and S. typhimurium. Inhibitory concentration $\mathrm{IC}_{50} \mathrm{was}_{\mathrm{s}}$ determined in S. typhimurium infected Caco-2 cells. Pharmacokinetics parameters and biodistribution study (in heart, liver, kidney and brain) of LFX and selected novel salts using $1 \mathrm{CBM}$ peroral Balb/c mice model was conducted. These salts have shown significant improvement in $\mathrm{MIC}$ and $\mathrm{IC}_{50}$ then LFX. So, these salts are more potent than pure drug. These salts are more water soluble, and we have seen this effect in the pharmacokinetic parameters like absorbance, plasma half lifetime, $\mathrm{T}_{\max }, \mathrm{C}_{\max }$, bioavailability, elimination rate constant and clearance of salts. Significant results of our study will be presented.
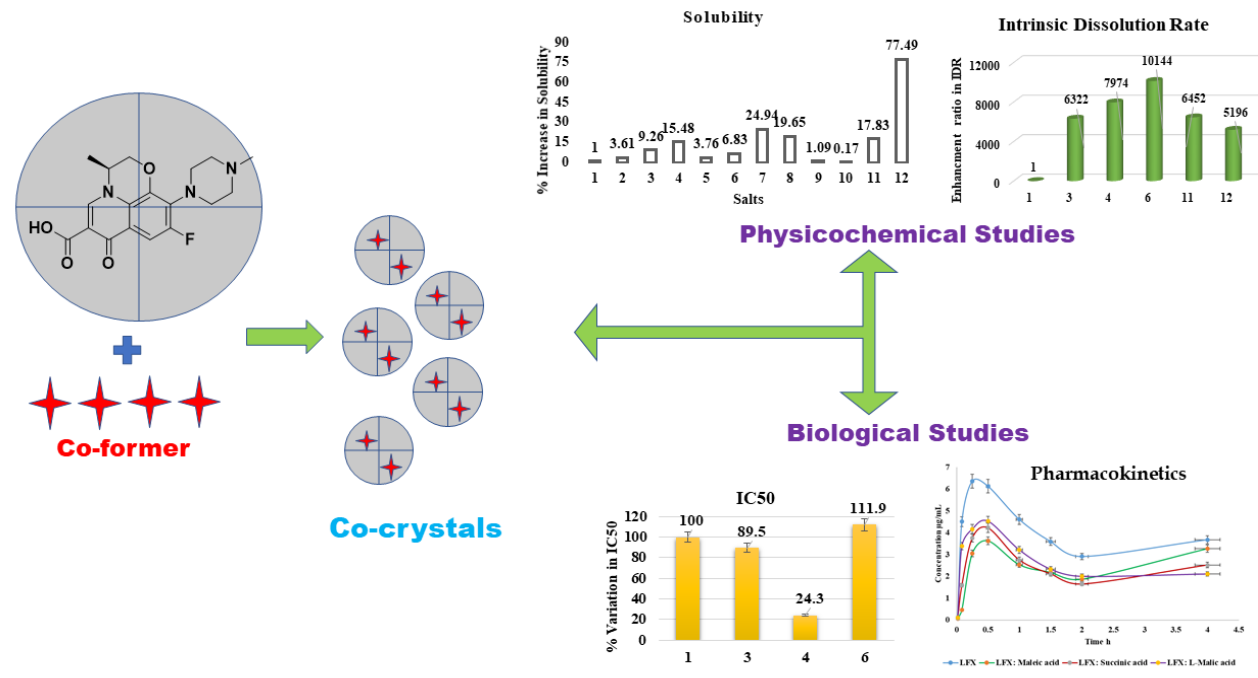

Figure 1. Graphical representation of Abstract

[1] Brittain, H. (2012). Cryst. Growth Des. 12, 5823

[2] Jones, W., Motherwell, W. D. S., Trask, A. V. (2006). MRS Bull. 31, 875.

[3] Joshi, M., Choudhury, A. R. (2018). ACS Omega. 3, 2406.

[4] Davis, R.; Bryson, H. M. (1994) Drugs, 47, 677.

Keywords: Cocrystallization; Solubility; Cell-line Assay; Pharmacokinetics; Biodistribution

Acta Cryst. (2021), A77, C877 